\title{
ALMOST CONVERGENCE AND THE BUCK-POLLARD PROPERTY
}

\section{G. M. PETERSEN}

Suppose that $\left\{S_{n}\right\}$ is a real sequence and $x$ a real number in the interval $0<x \leqq 1$. Representing $x$ by a nonterminating binary decimal expansion, we shall denote by $\left\{S_{n}(x)\right\}$ the subsequence obtained from $\left\{S_{n}\right\}$ by omitting $S_{k}$ if and only if there is a 0 in the $k$ th decimal place in the expansion of $x$. With this correspondence, it is then possible to speak of a "set of subsequences of the first category" an everywhere dense set of subsequences and so on.

For $(C, 1)$ summability, Buck and Pollard [1] have proved

THEOREM 1. If $\left\{S_{n}\right\}$ is $(C, 1)$ summable to $S$, and $\sum_{1}^{\infty} S_{k}^{2} / k^{2}$ converges, then almost all subsequences are $(C, 1)$ summable to $S$.

If $\left\{S_{n}\right\}$ is divergent, and $A=\left(a_{m n}\right)$ a regular matrix, a theorem of Keogh and Petersen [3] states the set of $A$-summable subsequences is of the first category. There are also results of a different nature due to Hill [2].

We now turn to the method of almost convergence which has been investigated by Lorentz. A bounded sequence $\left\{s_{n}\right\}$ is almost convergent if and only if

$$
\lim _{p \rightarrow \infty} \frac{1}{p} \sum_{n+1}^{n+p} s_{k}=s
$$

uniformly in $n$. We prove

THEOREM 2. There exist divergent sequences $\left\{s_{n}^{1}\right\}$ and $\left\{s_{n}^{2}\right\}$ such that $\left\{s_{n}^{1}\right\}$ and almost all of its subsequences are almost convergent and $\left\{s_{n}^{2}\right\}$ is almost convergent but almost all of its subsequences are not almost convergent.

Proof. For $\left\{s_{n}^{2}\right\}$ consider the sequence $1,0,1,0,1,0, \cdots$ Let $x$ be a normal number of base 2 ; for definition and theorems on normal numbers see Niven [6]. Given $x$, for every $p$ there exists a subsequence of the integers $\left\{n_{\nu}\right\}, \nu=1,2,3, \ldots$ (the terms of $\left\{n_{\nu}\right\}$ will depend on the choice of $p$ ) such that if $x=. x_{1} x_{2} \ldots$

$$
x_{k}=1, \quad n_{\nu} \leqq k \leqq n_{\nu}+2 p .
$$

Presented to the Society, January 22, 1959 under the title L-regular matrices; received by the editors November 26, 1958 and, in revised form, January 13, 1959, February 16, 1959, April 21, 1959, and July 2, 1959. 
Thus, the number $x$ has arbitrarily large blocks of 1's in its binary expansion and $\left\{s_{n}^{2}(x)\right\}$ has, correspondingly, large blocks of terms identical with large blocks of terms of $s_{n}^{2}$. In general the first term of such a block will correspond to different subscripts in the sequences $\left\{s_{n}^{2}(x)\right\}$ and $\left\{s_{n}^{2}\right\}$. More explicitly, for each $\nu=1,2,3, \cdots$ there is an $r_{\nu}, r_{\nu}=r_{\nu}(x)$ where $s_{r_{\nu}}^{2}(x)=s_{n_{\nu}}^{2}$ and

$$
\frac{1}{2 p} \sum_{r_{\nu}+1}^{r_{v}+2 p} s_{k}^{2}(x)=\frac{1}{2}, \quad \nu=1,2,3, \cdots
$$

Given $x$, for every $p$ there exists a subsequence of the integers $\left\{n_{\mu}\right\}, \mu=1,2,3, \cdots$ (again the terms of $\left\{n_{\mu}\right\}$ will depend on the choice of $p$ ) such that

$$
\begin{aligned}
& x_{k}=1, \quad k=n_{\mu}, n_{\mu}+2, \cdots, n_{\mu}+4 p, \\
& x_{k}=0, \quad k=n_{\mu}+1, \cdots, n_{\mu}+4 p+1 .
\end{aligned}
$$

Hence for each $\mu=1,2,3, \cdots$ there is an $r_{\mu}, r_{\mu}=r_{\mu}(x)$ where $s_{r_{\mu}}^{2}(x)$ $=s_{n_{\mu}}^{2}$ and either

$$
\frac{1}{2 p} \sum_{r_{\mu}+1}^{r_{\mu}+2 p} s_{k}^{2}(x)=0 \quad \text { or } \quad \frac{1}{2 p} \sum_{r_{\mu}+1}^{r_{\mu}+2 p} s_{k}^{2}(x)=1 .
$$

In any case

$$
\sup _{n} \frac{1}{2 p} \sum_{n+1}^{n+2 p} s_{k}^{2}(x)-\inf _{n} \frac{1}{2 p} \sum_{n+1}^{n+2 p} s_{k}^{2}(x) \geqq \frac{1}{2} .
$$

As the sequences $\left\{n_{\nu}\right\}$ and $\left\{n_{\mu}\right\}$ can be found for each $2 p$, it is clear

$$
\lim _{p \rightarrow \infty} \frac{1}{p} \sum_{n+1}^{n+p} s_{k}^{2}(x)
$$

does not exist uniformly in $n$ and the sequence is not almost convergent. Since almost all $x$ are normal, almost all subsequences are not almost convergent.

For $\left\{s_{n}^{1}\right\}$, let $s_{n}^{1}=0, n \neq \nu^{5}$, and $s_{\nu^{1}}^{1}=1$. Let $\left\{n_{k}\right\}$ be such a sequence that

$$
\lim _{k \rightarrow \infty} \frac{n_{k}-n_{k+1}}{n_{k}}=0 .
$$

We define $x$ by $x_{k}=1, k=n_{1}, n_{2}, \cdots, x_{k}=0$ otherwise, then $\left\{s_{n}^{1}(x)\right\}$ has at least $n^{3}$ zeros between its $n$th and its $(n+1)$ th one. Such a sequence is almost convergent to zero, see Lorentz [4, p. 171]. Hence, almost all subsequences of $\left\{s_{n}^{1}\right\}$ are almost convergent to zero as is $\left\{s_{n}^{1}\right\}$ itself $\longrightarrow$ see [8]. 
2. In this section we suppose that $A=\left(a_{m n}\right)$ is a strongly regular matrix, a matrix that sums every almost convergent sequence. A necessary and sufficient condition that a regular matrix be strongly regular is that

$$
\lim _{m \rightarrow \infty} \sum\left|a_{m n}-a_{m n+1}\right|=0,
$$

see Lorentz [4, p. 176]. The intersection of the summability fields for all strongly regular matrices is the set of almost convergent sequences [7]. On the other hand the intersection of any finite or countably infinite set of summability fields of strongly regular matrices contains a sequence that is not almost convergent, [4, p. 187].

For each $m, n=1,2, \cdots$ let

$$
t_{n}^{(m)}=\frac{1}{m}\left(s_{n+1}+\cdots+s_{n+m}\right) .
$$

Then almost convergence of $\left\{s_{n}\right\}$ means the uniform convergence in $n$ of the transforms: i.e. $\left|t_{n}^{(m)}-s\right|<\epsilon$ for $m \geqq M$ and all $n=1,2,3, \cdots$. We can arrange these transforms in an array, the $m$ th row of the array consisting of the transforms $t_{n}^{(m)}, n=1,2,3, \cdots$. Let $a$ be the family of matrices that can be developed by selecting transforms from the array for rows of the matrix. No more than a finite number of transforms are to be taken from each row of the array. The family $a$, for example, contains the $(C, 1)$ method-selecting for each row of the matrix the first transform in each row of the array. It is also clear that each member of $Q$ is strongly regular. If a matrix $A=\left(a_{m n}\right)$ sums all bounded sequences that are summed by $B=\left(b_{m n}\right), A$ is said to be $b$-stronger than $B$. By the iteration of $A$ with $B$, we mean the matrix $C=\left(c_{m n}\right)$, where

$$
c_{m n}=\sum a_{m i} b_{i n}
$$

$A, B$, and $C$, being row finite.

THEOREM 3. If $B$ is strongly regular, there is a matrix $A, A \in Q$ such that $B$ is b-stronger than $A$.

Proof. The matrix $B$ is strongly regular, if and only if there exists a function $\Omega(n)$ strictly increasing to infinity such that

$$
\lim _{m \rightarrow \infty} \sum \Omega(n)\left|b_{m n}-b_{m n+1}\right|=0 .
$$

The function $\Omega(n)$ is called a summability function for $B,[\mathbf{5}]$. We 
further construct the increasing integer valued functions $\lambda(m)$ and $\mu(m)$ and the sequence $\left(m_{k}\right)$ so that

$$
\sum_{1}^{\lambda(m)}\left|b_{m n}\right|<\epsilon_{m}, \quad \sum_{\mu(m)+1}^{\infty}\left|b_{m n}\right|<\epsilon_{m}
$$

and $\lim _{m \rightarrow \infty} \epsilon_{m}=0, \lambda\left(m_{k}\right)=\mu\left(m_{k-1}\right)$. In order to have $\left(m_{k}\right)$ well defined, we shall assume $\lambda(m) \leqq \lambda(m-1)+1$, for all $m=2,3, \cdots$. The matrix $B^{\prime}=\left(b_{m n}^{\prime}\right), b_{m n}^{\prime}=b_{m n}, \lambda(m) \leqq n \leqq \mu(m), b_{m n}^{\prime}=0$ elsewhere is $b$ equivalent to $B$. Suppose

$$
\tau_{k}=\frac{1}{r_{\nu}}\left(s_{k+1}+\cdots+s_{k+r_{\nu}}\right),
$$

for all $\lambda\left(m_{\nu}\right)<k \leqq \lambda\left(m_{\nu+1}\right)$, where $r_{\nu}$ shall be defined presently. Now if we iterate $B^{\prime}$ with $A, A$ being the matrix defined by the transforms $\tau_{k}, B^{\prime} \cdot A=C$, then if $\lambda\left(m_{v}\right)+r_{\nu}+1 \leqq n \leqq \lambda\left(m_{v+1}\right)+1$

$$
c_{m n}=\sum_{n-r_{\nu}}^{n-1} b_{m i}^{\prime} \frac{1}{r_{\nu}} \text {. }
$$

If we let

$$
d_{m n}=\sum_{n-r_{\nu}-1}^{\lambda\left(m_{\nu}\right)} b_{m i}^{\prime} \frac{1}{r_{\nu-1}} \text { and } d_{m n}^{\prime}=\sum_{\lambda\left(m_{\nu}\right)+1}^{n} b_{m i}^{\prime} \frac{1}{r_{\nu}},
$$

then for $\lambda\left(m_{\nu}\right)+1 \leqq n \leqq \lambda\left(m_{\nu}\right)+r_{\nu}$ we have $c_{m n}=d_{m n}+d_{m n}^{\prime}$ if $n-r_{\nu-1}$ $\leqq \lambda\left(m_{v}\right)$ and $c_{m n}=d_{m n}^{\prime}$ if $n-r_{v-1}>\lambda\left(m_{v}\right)$. If $\lambda\left(m_{v}\right)+r_{\nu}+1 \leqq n \leqq \mu(m)$ and $m_{\nu-1} \leqq m \leqq m_{\nu}$, then

$$
\begin{aligned}
\left|b_{m n}^{\prime}-c_{m n}\right| & =\left|b_{m n}^{\prime}-\frac{1}{r_{\nu}} \sum_{n-r_{\nu}}^{n-1} b_{m i}\right| \leqq \frac{1}{r_{\nu}} \sum_{n-r_{\nu}}^{n-1}\left|b_{m n}-b_{m i}\right| \\
& \leqq \frac{1}{r_{\nu}} \sum_{n-r_{\nu}}^{n-1} \sum_{j=i}^{n-1}\left|b_{m j}-b_{m, j+1}\right| \\
& \leqq \frac{1}{r_{\nu}} \sum_{n-r_{\nu}}^{n-1} r_{\nu}\left|b_{m i}-b_{m, i+1}\right|=\sum_{n \rightarrow r_{\nu}}^{n-1}\left|b_{m i}-b_{m, i+1}\right| .
\end{aligned}
$$

Similar calculations for $\lambda\left(m_{\nu}\right)+1 \leqq n \leqq \operatorname{Max}\left[\lambda\left(m_{\nu}\right)+r_{\nu}, \mu(m)\right], m_{\nu-1}$ $\leqq m \leqq m_{\nu}$, yield

$$
\left|b_{m n}^{\prime}-c_{m n}\right| \leqq 2 \sum_{n-r_{\eta}}^{n-1}\left|b_{m i}-b_{m, i+1}\right|
$$

providing $r_{\nu} \geqq r_{\nu-1}$. If $\left|s_{n}\right| \leqq M$ and $m_{\nu-1} \leqq m \leqq m$. 


$$
\begin{aligned}
& \left|\sum_{1}^{\infty}\left(b_{m n}^{\prime}-c_{m n}\right) s_{n}\right| \\
& \quad \leqq 2 M\left[\sum_{\lambda(m)}^{\left.\mu(m)^{-1}\right)} \sum_{n-r_{\nu-1}}^{n-1}\left|b_{m i}-b_{m i+1}\right|+\sum_{\lambda\left(m_{\nu}\right)+1}^{\mu(m)} \sum_{n-r_{\nu}}^{n-1}\left|b_{m i}-b_{m i+1}\right|\right] \\
& \quad \leqq 2 M\left[\sum_{\lambda(m)}^{\mu\left(m_{\nu}-1\right)} r_{\nu-1}\left|b_{m n}-b_{m n+1}\right|+\sum_{\lambda\left(m_{\nu}\right)+1}^{\mu(m)} r_{\nu}\left|b_{m n}-b_{m n+1}\right|\right] .
\end{aligned}
$$

Let $f(n)=r_{\nu}, \lambda\left(m_{\nu}\right)+1 \leqq n \leqq \mu\left(m_{\nu}\right)$. If $\Omega(n)$ is a summability function of $B$, we can have $r_{\nu}$ increase slowly enough with $\nu$ that $f(n) \leqq \Omega(n)$ for all $n$. It then follows that

$$
\lim _{m \rightarrow \infty} \sum f(n)\left|b_{m n}-b_{m n+1}\right|=0
$$

From our previous considerations we have for $\left|s_{n}\right| \leqq M$ :

$$
\left|\sum\left(b_{m n}^{\prime}-c_{m n}\right) s_{n}\right| \leqq 2 M \sum f(n)\left|b_{m n}-b_{m n+1}\right|
$$

so that

$$
\lim _{m \rightarrow \infty}\left|\sum\left(b_{m n}^{\prime}-c_{m n}\right) s_{n}\right|=0
$$

This means that $B^{\prime}$ and $B^{\prime} \cdot A$ are $b$-equivalent and since $B^{\prime} \cdot A$ is $b$-stronger than $A, B^{\prime}$ is $b$-stronger than $A$. This completes our proof.

3. A regular matrix $A=\left(a_{m n}\right)$ is one which transforms convergent sequences into sequences convergent to the same sum. We shall call the matrix $L$-regular if it is regular and transforms almost convergent sequences into sequences that are almost convergent to the same sum. The strongly regular matrices are $L$-regular, for example, as they transform the sequences that are almost convergent to a sum $s$ into sequences that converge to $s$. It is also clear that if $A$ and $B$ are $L$-regular, then $A \cdot B$ is $L$-regular. If $A$ is not $L$-regular, then it must transform an almost convergent sequence into one that is almost convergent to a different sum or one that is not almost convergent.

Lemma 1. If $B$ is a regular matrix, then $B$ is L-regular if and only if $A \cdot B$ is strongly regular for every $A$ in $Q$.

Proof. Every $A$ in $Q$ is strongly regular and hence sums every almost convergent sequence to its almost convergent sum. Hence, if $B$ is $L$-regular, it is clear that $A \cdot B$ will be strongly regular, see [4, p. 169]. On the other hand if $B$ transforms a sequence $\left\{s_{n}\right\}$ almost convergent to $s$ into a sequence $\left\{s_{n}^{\prime}\right\}$ almost convergent to $s^{\prime}$, then 
$A \cdot B$ sums $\left\{s_{n}\right\}$ to $s^{\prime}$ and $A \cdot B$ is inconsistent with almost convergence. Hence, see $[4$, p. 176$], A \cdot B$ cannot be strongly regular. If $\left\{s_{n}\right\}$ is an almost convergent sequence and its transform $\left\{s_{n}^{\prime}\right\}$ is not almost convergent, then a matrix of $a$ can be found that sums $\left\{s_{n}^{\prime}\right\}$ to any value between

$$
\inf \lim _{k \rightarrow \infty} \sup \frac{1}{p} \sum_{k}^{k+p} s_{n} \text { and } \sup _{k \rightarrow \infty} \operatorname{limf} \frac{1}{p} \sum_{k}^{k+p} s_{n} .
$$

Clearly not all $A \cdot B$ will sum $\left\{s_{n}\right\}$ to the proper sum. Hence not all $A \cdot B$ will be strongly regular. This completes our proof.

The quantity $k-\operatorname{Var}\left(A_{m}\right)$ will be defined by

$$
\begin{aligned}
k-\operatorname{Var}\left(A_{m}\right)=\sum_{n=1}^{\infty} \mid\left(a_{m n}+\cdots+a_{m+k-1, n}\right) & -\left(a_{m, n+1}+\cdots+a_{m+k-1, n+1}\right) \mid
\end{aligned}
$$

and $V(k)$ is defined by

$$
V(k)=\sup _{m} k-\operatorname{Var}\left(A_{m}\right) .
$$

The $1-\operatorname{Var}\left(A_{m}\right)$, for example, is given by

$$
1-\operatorname{Var}\left(A_{m}\right)=\sum_{n=1}^{\infty}\left|a_{m n}-a_{m, n+1}\right| .
$$

Let $S$ be the set of transforms

$$
v_{n}^{(m)}=\sum_{k=1}^{\infty} a_{m k}^{(n)} s_{k},
$$

then $S$ can be arranged in an array of transforms, the $m$ th row of the array consisting of the transforms $v_{n}^{(m)}, n=1,2,3, \cdots$. Let $\delta$ be the family of matrices that can be developed by selecting transforms from the array for rows of the matrix. No more than a finite number of transforms are to be taken from each row of the array. The array is said to sum a sequence $\left\{s_{n}\right\}$ to $s$ if for all $\epsilon>0$,

$$
\left|v_{n}^{(m)}-s\right|<\epsilon \quad \text { for } m \geqq m(\epsilon) \text { and all } n=1,2, \cdots .
$$

An array is called regular if it sums every convergent sequence to the sum it converges to, and strongly regular if it sums every almost convergent sequence to the sum it is almost convergent to. It can be shown, [7], that an array is regular if and only if every member of $s$ is regular and it is strongly regular if and only if every member of $s$ is strongly regular. Hereafter, we shall mean by $S$ the set of trans- 
forms arranged in the array described above. If the array is strongly regular or regular, we shall speak of $S$ as being strongly regular or regular.

LeMma 2. The array $S$ is strongly regular if and only if for all $\epsilon>0$,

$$
\sum_{k=1}^{\infty}\left|a_{m k}^{(n)}-a_{m, k+1}^{(n)}\right|<\epsilon \quad \text { for } m \geqq m(\epsilon) \text { and all } n=1,2, \cdots \text {. }
$$

Proof. An array $S$ is strongly regular if and only if every matrix of $\delta$ is strongly regular - see [7]. If the condition of the lemma is not satisfied, there is an infinite sequence $\left\{m_{\mu}\right\}$ and a corresponding $\left\{n\left(m_{\mu}\right)\right\}$ such that

$$
\sum\left|a_{m_{\mu} k}^{\left(n_{\mu}\right)}-a_{m_{\mu}, k+1}^{\left(n_{\mu}\right)}\right|>\epsilon
$$

for some $\epsilon$. A matrix made up from the rows $\left\{v_{n_{\mu}}^{\left(m_{\mu}\right)}\right\}$ is not therefore strongly regular. If the condition of the lemma is satisfied, it is plain that all the matrices of $\delta$ are strongly regular. This proves our assertion.

Theorem 4. $A$ regular matrix $B=\left(b_{m n}\right)$ is $L$-regular if and only if $V(k)=o(k)$.

Suppose $\tau_{m}=\sum b_{m n} s_{n}$, then the condition of Lemma 1 is equivalent to the statement that every matrix belonging to the family $\mathfrak{I}$ corresponding to the set $T$ of transforms $t_{n}^{(m)}$ be strongly regular, where

$$
t_{n}^{(m)}=\frac{1}{m} \sum_{n}^{n+m-1} \tau_{k}
$$

From Lemma 2 this will be the case if and only if

$$
\sum\left|a_{m k}^{(n)}-a_{m, k+1}^{(n)}\right|<\epsilon, \quad \text { for } m \geqq m(\epsilon) \text { and } n=1,2, \cdots,
$$

where $t_{n}^{(m)}=\sum a_{m k}^{(n)} s_{k}$. However

$$
a_{m k}^{(n)}=\frac{1}{m} \sum_{n}^{n+m-1} b_{i k}
$$

and

$$
\sum\left|a_{m k}^{(m)}-a_{m, k+1}^{(n)}\right|=\frac{1}{m} m-\operatorname{Var}\left(B_{n}\right) \leqq \frac{1}{m} V(m),
$$

and so our theorem is proved. 
4. We now shall give some examples of $L$-regular matrices. First, however, we shall prove a theorem concerning the matrices $C=\left(c_{m n}\right)$ where $c_{m n}=1 / k, m \leqq n \leqq m+k, c_{m n}=0$ elsewhere, $u_{m}=\sum c_{m n} s_{n}$. The reader can easily check that these matrices are all $L$-regular.

Theorem 5. Almost convergence is b-stronger than any one of the matrices just defined.

Proof. Consider the array $W$ where

$$
w_{n}^{(m)}=\frac{1}{m} \sum_{n+1}^{n+m} u_{r}
$$

since $C$ is $L$-regular, it follows as in Theorem 4 that the method defined by the array $W$ is strongly regular. Moreover for

$$
\begin{aligned}
m & >2 k \frac{1}{m}\left(s_{n+1}+\cdots+s_{n+m}\right)-\frac{1}{m} \sum_{n+1}^{n+m} u_{r} \\
& \leqq \frac{1}{m}\left[\sum_{n+1}^{n+k}\left|s_{r}\right|+\sum_{n+m-k}^{n+m}\left|s_{r}\right|\right],
\end{aligned}
$$

so that the difference converges to zero independently of $n$ if $\left|s_{r}\right| \leqq M$ and the method described by the array $W$ is $b$-equivalent to almost convergence. However, it is clear that this array sums any sequence for which $\left\{u_{m}\right\}$ converges. Hence almost convergence is $b$-stronger than the matrix $C$.

The matrices of Theorem 5 provide examples of $L$-regular matrices, not all nonstrongly regular $L$-regular matrices have their bounded convergence field contained by almost convergence, however. For example, consider the identity matrix $D=\left(d_{m n}\right), d_{m m}=1 ; d_{m n}=0$, $m \neq n$. Form the submatrix $D^{\prime}$ by retaining the rows

$$
\begin{array}{r}
k=1,2, \cdots, \nu^{4}-1, \nu^{4}+\nu+1, \cdots,(\nu+1)^{4}-1, \\
(\nu+1)^{4}+\nu+2, \cdots .
\end{array}
$$

This matrix is $L$-regular, but it sums the sequence $\left\{s_{n}\right\}$, where $s_{n}=1, \nu^{4} \leqq n \leqq \nu^{4}+\nu, s_{n}=0$ elsewhere, and this sequence is not almost convergent.

Finally we remark that the matrices of Theorem 5, cannot, after Theorem 2, have the Buck-Pollard property.

\section{REFERENCES}

1. R. C. Buck and H. Pollard, Convergence and summability properties of subsequences, Bull. Amer. Math. Soc. vol. 49 (1943) pp. 924-931. 
2. J. D. Hill, Remarks on the Borel property, Pacific J. Math. vol. 4 (1954) pp. 227-242.

3. F. R. Keogh and G. M. Petersen, A universal Tauberian theorem, J. London Math. Soc. vol. 33 (1958) pp. 121-123.

4. G. G. Lorentz, A contribution to the theory of divergent sequences, Acta Math. vol. 80 (1948) pp. 167-190.

5. - Direct theorems on methods of summability, Canad. J. Math. vol. 1 pp. 305-319, especially p. 307.

6. I. Niven, Irrational numbers, Carus monographs, no. 11, 1956, 164 pp., especially pp. $98-113$.

7. G. M. Petersen, Almost convergence and uniformly distributed sequences, Quart. J. Math. Oxford Ser. (2) vol. 7 (1956) pp. 188-191.

8. Casper Goffman and G. M. Petersen, Submethods of regular matrix summability methods, Canad. J. Math. vol. 8 (1956) pp. 40-46, especially p. 44.

University of New MeXico 